\title{
RUNX3 plays a tumor suppressor role by inhibiting cell migration, invasion and angiogenesis in oral squamous cell carcinoma
}

\author{
WEI-NA ZHOU ${ }^{1,2^{*}}$, YI-FEI DU ${ }^{1,3^{*}}$, JIN BAI $^{4 *}$, XIAO-MENG SONG ${ }^{1,3}$, YANG ZHENG $^{1,3}$, \\ HUA YUAN $^{1,3}$, WEI ZHANG ${ }^{1,5}$, ZHENG-DONG ZHANG ${ }^{6}$ and YU-NONG WU ${ }^{1,3}$ \\ ${ }^{1}$ Jiangsu Key Laboratory of Oral Diseases, Nanjing Medical University; Departments of ${ }^{2}$ Polyclinic Dentistry and \\ ${ }^{3}$ Oral and Maxillofacial Surgery, Affiliated Hospital of Stomatology, Nanjing Medical University, Nanjing; \\ ${ }^{4}$ Jiangsu Center for the Collaboration and Innovation of Cancer Biotherapy, Cancer Institute, Xuzhou Medical University, \\ Xuzhou; ${ }^{5}$ Department of Pathology, Affiliated Hospital of Stomatology, Nanjing Medical University; \\ ${ }^{6}$ Department of Genetic Toxicology, The Key Laboratory of Modern Toxicology of Ministry of Education, \\ School of Public Health, Nanjing Medical University, Nanjing, Jiangsu, P.R. China
}

Received January 26, 2017; Accepted June 20, 2017

DOI: $10.3892 / o r .2017 .5857$

\begin{abstract}
Although aberrant expression of Runt-related transcription factor 3 (RUNX3) contributes to tumor progression and metastasis in a number of carcinomas, the status of RUNX3 and its correlation with prognosis in oral squamous cell carcinomas (OSCC) are still controversial. The aim of present study was to investigate the function of RUNX3 in OSCC and the underlying molecular mechanisms. Tissue microarray (TMA) consisting of 232 OSCC specimens was used to detect the expression of RUNX3 by immunohistochemistry method. The effects of RUNX3 restoration on OSCC cell migration and invasion were determined by wound-healing assay, migration and Matrigel cell invasion assays. The antiangiogenic role of RUNX3 was analyzed by testing proliferation and tube formation of human umbilical vascular endothelial cells (HUVECs) cultured with conditioned medium from RUNX3 transfected OSCC cell lines. The activities of MMP-9 and VEGF in RUNX3 transfected OSCC cell lines were examined by western blot and ELISA methods. RUNX3 expression was reduced in OSCC speci-
\end{abstract}

Correspondence to: Dr Zheng-Dong Zhang, Department of Genetic Toxicology, The Key Laboratory of Modern Toxicology of Ministry of Education, School of Public Health, Nanjing Medical University, 140 Hanzhong Road, Nanjing, Jiangsu 210029, P.R. China

E-mail: zdzhang@njmu.edu.cn

Dr Yu-Nong Wu, Jiangsu Key Laboratory of Oral Diseases, Nanjing Medical University, 136 Hanzhong Road, Nanjing, Jiangsu 210029, P.R. China

E-mail: yunongwu@aliyun.com

${ }^{*}$ Contributed equally

Key words: RUNX3, oral squamous cell carcinoma, tissue microarray, migration, invasion, angiogenesis mens and significantly associated with tumor size $(\mathrm{P}=0.002)$, lymph node statue $(\mathrm{P}=0.0036)$ and clinical stage $(\mathrm{P}=0.0001)$. Negative expression of RUNX3 correlated with worse 5-year overall and disease-specific survival rates $(\mathrm{P}=0.0348$ and $\mathrm{P}=0.0301$, respectively). Furthermore, we found that RUNX3 restoration suppressed cell migration and invasion through downregulating MMP-9 expression and secretion, and exerted antiangiogenic capability by inhibiting VEGF activity in HN6 and Cal27 cells. These findings suggested that RUNX3 played a tumor suppressor role in OSCC by inhibiting cell migration, invasion and angiogenesis, supporting that it could be a potential therapeutic target for OSCC.

\section{Introduction}

Oral squamous cell carcinoma (OSCC) accounts for $>90 \%$ of oral malignancies, with $\sim 250,000$ new cases each year worldwide $(1,2)$. Approximately two-thirds of these cases occur in developing countries, especially in the South and Southeast Asia. Despite consistent efforts of therapy development including surgery, chemotherapy and radiotherapy, the prognosis of OSCC is still poor because of tumor recurrence and metastasis (3). Hence, it is important to seek potential therapeutic biomarkers of OSCC, which are critical to early prediction and treatment evaluation (4).

Runt-related transcription factor 3 (RUNX3), one member of RUNX family of DNA-binding transcription factors, has been shown closely related to tumorigenesis (5). The inactivation of RUNX3 was observed in various human cancer types, such as esophageal (6), gastric (7), colorectal (8), lung (9) and breast cancer (10), caused by promoter hypermethylation and protein mislocalization. The restoration of RUNX3 could inhibit the metastasis and angiogenesis of many cancer cells in vitro (11-14). These studies supported that RUNX3 acted as a tumor suppressor. However, other studies provided evidence that RUNX3 may have an oncogenic role in head and neck squamous cell carcinoma (HNSCC) $(15,16)$ and skin cancers (15). 
In OSCC, the RUNX3 function as either an oncogene or a tumor suppressor gene is controversial. Tanji et al found higher labeling indexes (LIs) of RUNX3 expression in OSCC tissues compared with that in the normal epithelia, but they also pointed out that RUNX3 LIs correlated with the histological grades of OSCC, being the highest in the well differentiated OSCC (17). On the other hand, Gao et al discovered that the expression of RUNX3 protein was markedly reduced in OSCC specimens compared with the matched adjacent normal tissues. Furthermore, they found that only $14.7 \%$ of OSCC samples (22 of 150) showed a normal nuclear localization of RUNX3 protein (18). All these findings suggested the function of RUNX3 and the underlying mechanism in oral carcinogenesis are still unclear.

The objectives of the present study were to examine the RUNX3 staining in 232 OSCC samples using tissue microarray (TMA) technology and analyze its correlation with clinicopathological parameters. Also, we tried to clarify the potential molecular mechanisms.

\section{Materials and methods}

Ethics statement. This study was performed under a protocol approved by the Institutional Review Boards of Affiliated Stomatological Hospital of Nanjing Medical College and all examinations were performed after obtaining written informed consents.

Patients and samples. A total of 232 OSCC patients hospitalized in Department of Oral and Maxillofacial Surgery, Stomatological Hospital of Nanjing Medical College from January 2008 to January 2014 were included in this study. None of the patients received preoperative radiotherapy or chemotherapy. Each patient was pathologically diagnosed as squamous cell carcinoma and graded according to WHO criteria by two pathologists. All patients were followed up at least 24 months and the complete clinicopathological data including recurrence and metastasis were collected.

These OSCC samples were sent to Jiangsu Key Laboratory of Biological Cancer Therapy, Xuzhou Medical College (Xuzhou, China) to manufacture the OSCC TMA. The array dot diameter was $1.5 \mathrm{~mm}$, and each dot represented a tissue spot from one individual specimen that was selected and pathologically confirmed.

ImmunohistochemistryofOSCCTMA.Immunohistochemistry of OSCC TMA was performed with the streptavidin-peroxidase (Sp) method using a standard Sp kit (Zhongshan Biotech, Beijing, China). The TMA slide was incubated with monoclonal mouse anti-RUNX3 antibody (1:250, Abcam, USA) overnight at $4^{\circ} \mathrm{C}$, and diaminobenzidine (DAB; Zhongshan Biotech, China) was used to produce a brown precipitate. Negative controls were obtained by substituting primary antibodies with non-immune serum. The immunoreactivity was assessed blindly by two independent observers using Zeiss Imager Z1 microscope, and the image was collected by AxioCam MRc5 camera. The expression of RUNX3 was graded as positive when $5 \%$ of tumor cells showed immunopositivity, while the biopsies with $<5 \%$ were considered negative (18).
Cell lines and transfection. Human OSCC cell lines HN6 and Cal27 were purchased from the Shanghai Institute of Biochemistry and Cell Biology, Chinese Academy of Sciences (Shanghai, China). Human umbilical vascular endothelial cells (HUVECs) were obtained from KeyGen Biotech (Nanjing, China). HN6 and Cal27 cells were cultured in RPMI-1640 medium supplemented with $10 \%$ fetal calf serum (Invitrogen, Shanghai, China), and HUVECs were cultured in DMEM medium supplemented with $10 \%$ fetal calf serum. Cells were placed at $37^{\circ} \mathrm{C}$ in humidified incubator with $95 \%$ air, $5 \% \mathrm{CO}_{2}$.

The pFlag-control and pFlag-RUNX3 expression plasmids were obtained from Jiangsu Key Laboratory of Biological Cancer Therapy, Xuzhou Medical College (Xuzhou, China). Transfection of the pFlag-control and the pFlag-RUNX3 plasmids into HN6 and Cal27 was carried out using Lipofectamine 2000 transfection reagent (Invitrogen) following the manufacturer's protocol.

Scratch wound healing assay. Cells were seeded in 6-well plates at a density of $5 \times 10^{4}$ cells/well and cultured to confluence. The cell monolayer was serum-free starved overnight. Confluent cell monolayer was then scraped with a yellow pipette tip for scratch wounds and washed twice with PBS. After 24-h incubation, the cell images were captured in the same position. The wound areas were evaluated by AxioVision 4.8 software.

Migration assay. Cell migration was determined using Transwell migration assay ( $8-\mu \mathrm{m}$ pore size; Cell Biolabs). Cells were seeded into the upper chamber in serum-free medium at a density of $5 \times 10^{4}$. After a 12 -h incubation at $37^{\circ} \mathrm{C}$, cells in the upper chamber were carefully removed with a cotton swab. Cells traversed the membrane were fixed in methanol and stained with leucocrystal violet. Images were taken with an inverted microscope. Five random selected fields of view were captured and the invasive cells were counted.

Invasion assay. The Transwell filter inserts were coated with Matrigel (BD Biosciences, NJ, USA). Transfected 0.5x $10^{5} \mathrm{HN} 6$ cells and $1 \times 10^{5}$ Cal 27 cells were seeded in serum-free medium in the upper chamber. After a $24-\mathrm{h}$ incubation at $37^{\circ} \mathrm{C}$, cells at the top of the Matrigel were gently removed with a cotton swab. Invasive cells at the bottom of Matrigel were fixed in methanol, stained with leucocrystal violet and counted.

Cell proliferation assay. Transfected HN6 and Cal27 cells $\left(1 \times 10^{6}\right)$ were cultured in 6-well plate with serum-free medium for $24 \mathrm{~h}$. The medium was collected as a conditioned medium. Cellular proliferation was performed using Cell counting kit-8 (CCK-8) purchased from Beyotime Institute of Biotechnology (Nanjing, China). Briefly, 2x10 4 HUVECs were suspended in conditioned medium or vehicle control and seeded at a density of $2 \times 10^{4}$ per well in a 96-well plate. After incubation at $37^{\circ} \mathrm{C}$ for $24 \mathrm{~h}$, cell proliferation was detected according to the manufacturer's instructions.

Endothelial cell tube formation assay. HUVECs were starved overnight and then seeded at a density of $2 \times 10^{4}$ per well in a 48-well plate pre-coated with Matrigel. After incubation at $37^{\circ} \mathrm{C}$ for $30 \mathrm{~min}$, the medium was replaced with conditioned 
medium or vehicle control. After a 24-h incubation, three random selected fields of view were captured. Quantified evaluation of tube formation was obtained from measuring the length of tube-like structures. The experiments were repeated three times.

Western blot analysis. Cells were washed with PBS and lysed in radioimmunoprecipitation lysis buffer (Beyotime, China) containing $1 \mathrm{mM}$ phenylmethylsulfonyl fluoride based on the manufacturer's instructions. The cell protein $(10 \mu \mathrm{g})$ was separated on a $12 \%$ SDS-polyacrylamide gel. The protein was then transferred to nitrocellulose membrane and incubated overnight at $4^{\circ} \mathrm{C}$ with the following antibodies: mouse anti-RUNX3 (1:2,000, Abcam), mouse anti-VEGF (1:1,000, Abcam), rabbit anti-MMP9 and mouse anti- $\beta$-actin $(1: 1,000$, Boster Biotechnology, China). Membranes were then washed in PBS and incubated with secondary antibody (goat anti-rabbit and goat anti-mouse IgG) for $2 \mathrm{~h}$ and then washed in PBST (PBS containing Tween-20) at room temperature. After that, the membrane was stained by coloration fluid which contains $10 \mathrm{ml}$ alkaline phosphatase buffer, $33 \mu \mathrm{l} \mathrm{BCIP}$, and $66 \mu \mathrm{l} \mathrm{NBT}$. Finally, the protein bands were detected using SuperSignal West Pico Chemiluminescent Substrate (Thermo Fisher Scientific, Waltham, MA, USA) and exposed to Kodak X-ray film. Three independent trials of each experiment were carried out.

ELISA for MMP-9 and VEGF. HN6 and Cal27 cells were seeded in 6-well plates at a density of $1 \times 10^{6}$ cells per well. Then, cells were transfected with pFlag-control and pFlag-RUNX3 with serum-free medium. The supernatants were collected $24 \mathrm{~h}$ after transfection. MMP-9 and VEGF concentration were determined using Quantikine ELISA kits according to the manufacturer's instructions (R\&D Systems, MN, USA).

Statistical analysis. Statistical analysis was performed with SPSS 20 software (SPSS Inc., IL, USA) and data were expressed as the mean $\pm \mathrm{SD}$. The associations between RUNX3 staining and the clinicopathologic parameters were evaluated by two-sided Fisher's exact and $\chi^{2}$ tests. The correlation between statue of RUNX3 expression and patient's survival was analyzed by Kaplan-Meier survival analysis. For migration and invasion assays, endothelial cell tube formation and CCK-8 cell proliferation assays, and data of western blotting and ELISA, Student's t-test was used. Differences were considered significant when $\mathrm{P}<0.05$.

\section{Results}

Aberrant expression of RUNX3 protein in OSCC. We first determined whether RUNX3 expression was changed in human OSCC. Immunohistochemistry of the OSCC TMA was performed to detect the expression of RUNX3 protein and the distribution of RUNX3-positive cells. We observed RUNX3 staining displayed different patterns: 93 (40.1\%) cases presented with positive nuclear staining and 85 (36.6\%) cases with positive cytoplasmic staining, and only 54 (23.2\%) cases showed underexpression of RUNX3 protein with undetectable or low levels of staining (Fig. 1A). The cytoplasmic staining, termed as 'RUNX3 mislocalization' in the cytoplasm,
Table I. RUNX3 expression and clinicopathological characteristics of 232 OSCCs.

RUNX3 staining

Variables Negative (\%) Positive (\%) Total P-value

Age

$\begin{array}{lrrrr}\geq 60 \text { years } & 75(60.0) & 50(40.0) & 125 & 1.0 \\ <60 \text { years } & 64(59.8) & 43(40.2) & 107 & \\ \text { Sex } & & & & \\ \text { Male } & 82(58.2) & 59(41.8) & 141 & 0.5833 \\ \text { Female } & 57(62.6) & 34(37.4) & 91 & \\ \text { Tumor size } & & & & \\ \text { T1 and T2 } & 107(55.4) & 86(44.6) & 193 & 0.0021 \\ \text { T3 and T4 } & 32(82.2) & 7(17.8) & 39 & \end{array}$

Lymph node

status

$\begin{array}{lrrrr}\text { N0 } & 62(48.4) & 66(51.6) & 128 & <0.0001 \\ \text { N1, 2 and 3 } & 77(74.0) & 27(26.0) & 104 & \\ \text { Invasion status } & & & & \\ \text { Yes } & 128(61.8) & 79(38.2) & 207 & 0.1290 \\ \text { No } & 11(44.0) & 14(56.0) & 25 & \end{array}$

Histological

grade

$\begin{array}{lrrrr}\text { I } & 70(53.8) & 60(46.2) & 130 & 0.1030 \\ \text { II } & 53(67.9) & 25(32.1) & 78 & \\ \text { III } & 16(66.7) & 8(33.3) & 24 & \\ \text { Clinical stage } & & & & \\ \text { I } & 33(43.4) & 43(56.6) & 76 & 0.0001 \\ \text { II } & 19(47.5) & 21(52.5) & 40 & \\ \text { III } & 40(72.7) & 15(27.3) & 55 & \\ \text { IV } & 46(77.0) & 14(23.0) & 61 & \end{array}$

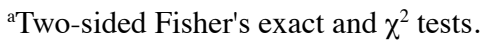

suggested RUNX3 protein was inactive in a non-functional form as a tumor suppressor $(7,10)$. Both underexpression and mislocalization of RUNX3 protein were aberrant and represented RUNX3 gene impairment in cancer cells; hence we analyzed these two patterns together as negative expression.

Correlation of RUNX3 expression with clinicopathological parameters. Based on TMA results, we further investigated the relationship between RUNX3 expression and the clinicopathological parameters of OSCC. We found that positive expression of RUNX3 was more frequent in T1/T2 compared with $\mathrm{T} 3 / \mathrm{T} 4$ cases $(\mathrm{P}=0.0021)$. Also, we found that negative expression of RUNX3 was significantly correlated with lymph node statue and clinical stage $(\mathrm{P}<0.0001$ and $\mathrm{P}=0.000$, respectively). The correlations between expression of RUNX3 with other clinicopathological variables, including patient age, 
A
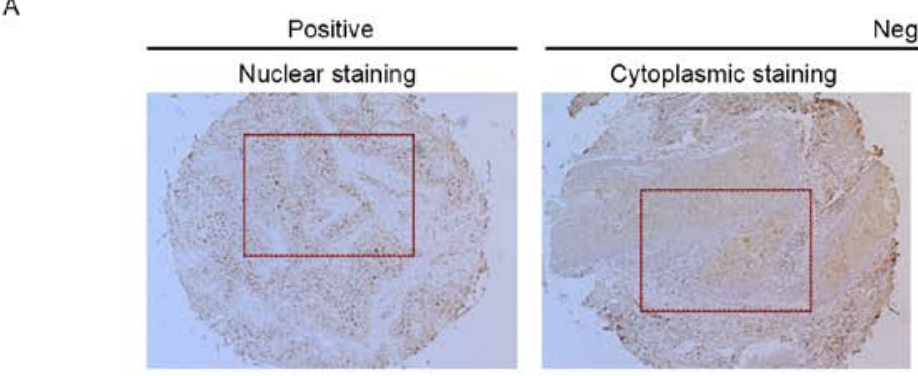

Negative
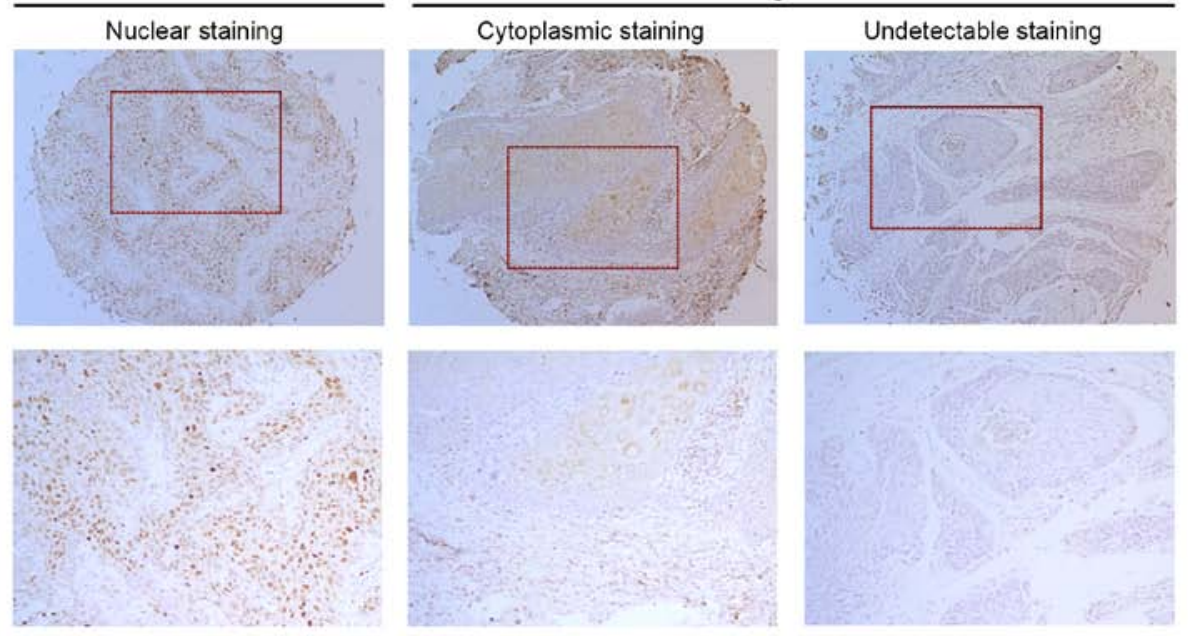

B
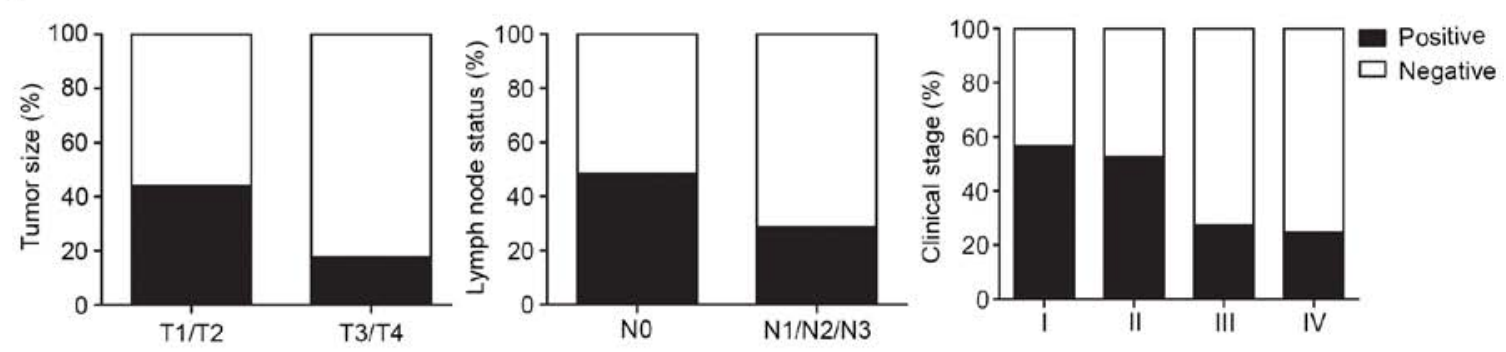

C

IIIV OSCC
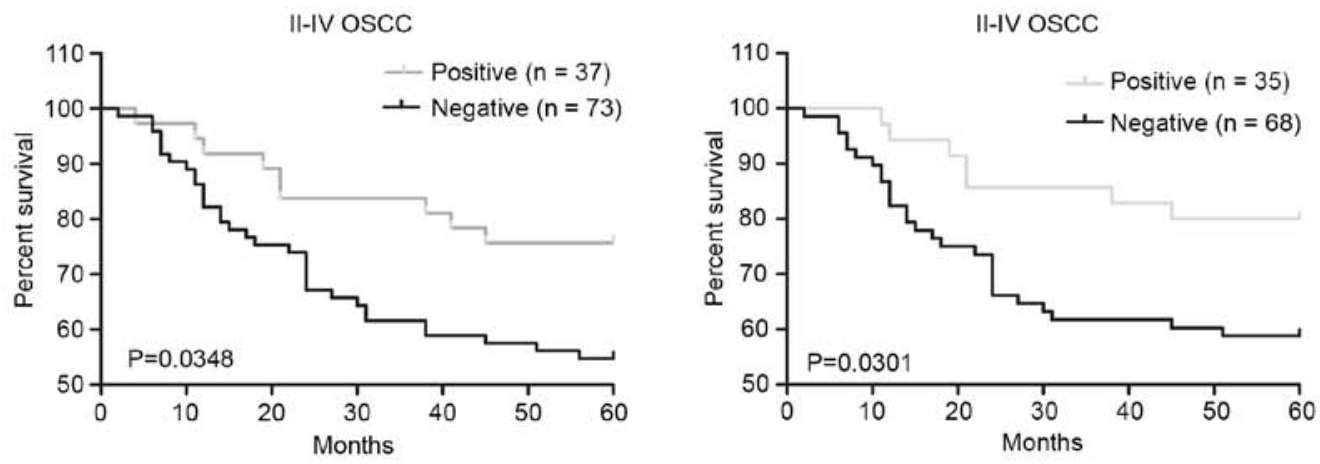

Figure 1. Negative expression of RUNX3 is associated with tumor size, lymph node metastasis and clinical stage, and worsens patients' survival. (A) Representative images of RUNX3 immunohistochemical staining in OSCC tissues. Left panel, positive expression of nuclear staining in OSCC tissue. Middle panel, negative expression of cytoplasmic staining in OSCC tissue. Right panel, negative expression of undectectable staining in OSCC tissue. Top panel, magnification, $x 100$. Bottom panel, magnification, $x 200$. (B) Reduced RUNX3 expression correlates with high tumor size $\left(\mathrm{P}=0.0021, \chi^{2}\right.$ test), lymph node metastasis $\left(\mathrm{P}<0.0001, \chi^{2}\right.$ test $),(\mathrm{P}=0.000$, by two-sided Fisher's exact test). (C) Negative RUNX3 expression correlates with a poorer 5 -year overall survival ( $\mathrm{P}=0.0349, \log$-rank test) and 5-year disease-specific survival $(\mathrm{P}=0.0301, \log$-rank test) for 110 II-IV OSCC patients.

sex, invasion stage and histological grade, were not significant (Fig. 1B and Table I).

Negative expression of RUNX3 correlates with poor patient survival. We then analyzed whether the RUNX3 expression was associated with the survival of patients by Kaplan-Meier survival analysis. The cases of clinical stage I were excluded because of favorable prognosis and then 110 cases (among 156 cases of clinical stage II-IV) with at least 60 months follow-up were chosen for prognosis analysis. Our data revealed that negative expression of RUNX3 in OSCC correlated with both 5-year overall and disease-specific patient survival $(\mathrm{P}=0.0348$ and $\mathrm{P}=0.0301$, respectively, log-rank test; Fig. 1C).
Inhibition of cells migration and invasion in RUNX3 transfected OSCC cells in vitro. To determine the effects of RUNX3 restoration on OSCC cell migration and invasion, we transiently transfected HN6 and Cal27 cells with pFlag-control and pFlag-RUNX3 plasmids. Twenty-four hours after transfection, RUNX3 protein was significantly overexpressed in cancer cells (Fig. 2A and B). The protein detection of RUNX3 by western blotting showed two bands, and this could be due to protein mislocalization, which was reported in a previous study (7). The results of wound healing and Transwell assays showed that RUNX3 tansfected cells showed less migration ability compared with vehicle control (Fig. 2C-E). Moreover, the invasion assay proved that RUNX3 restoration inhibited 
A

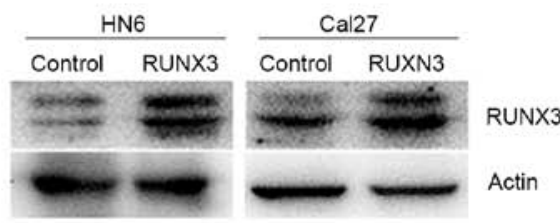

c
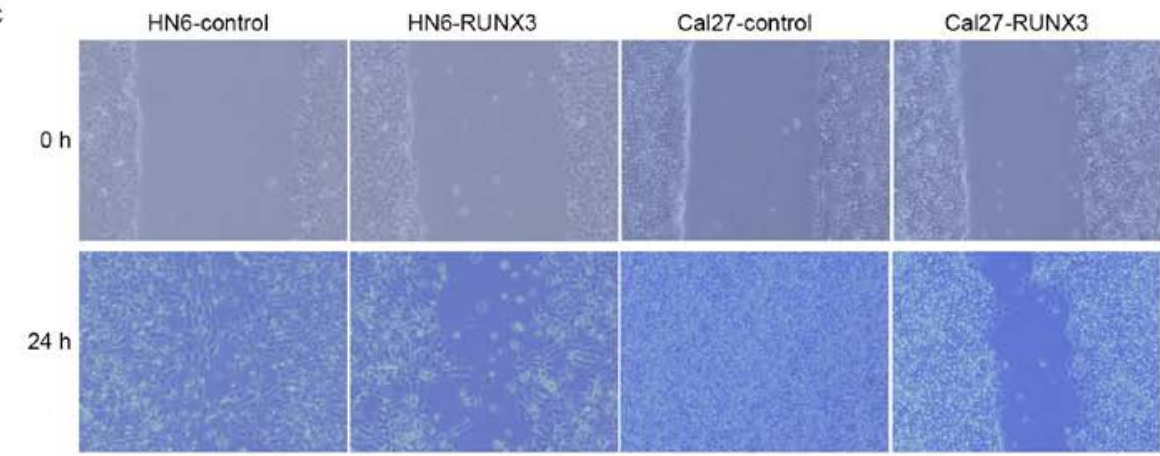

D
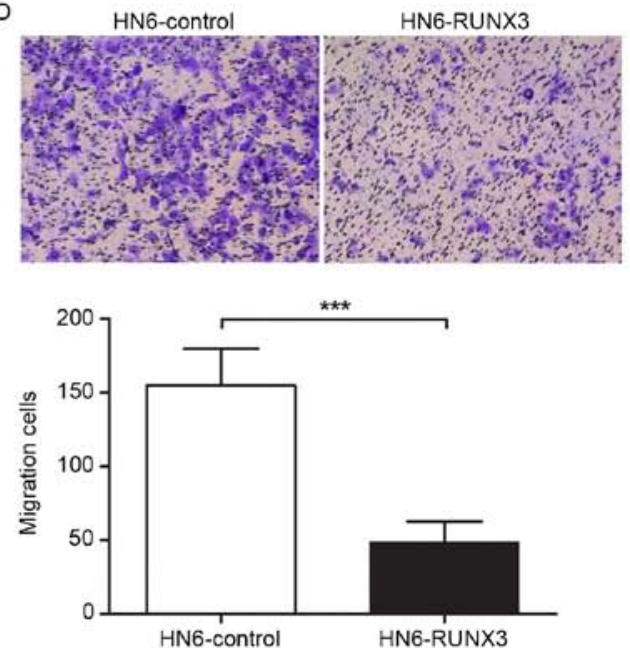

F
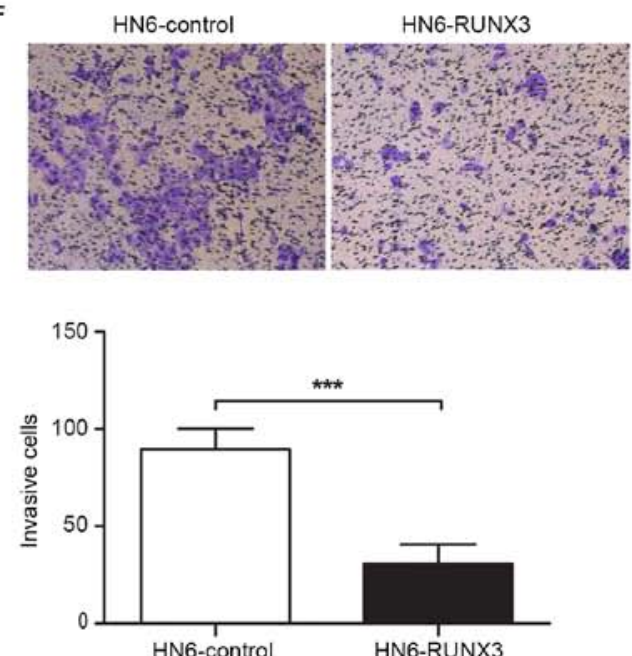

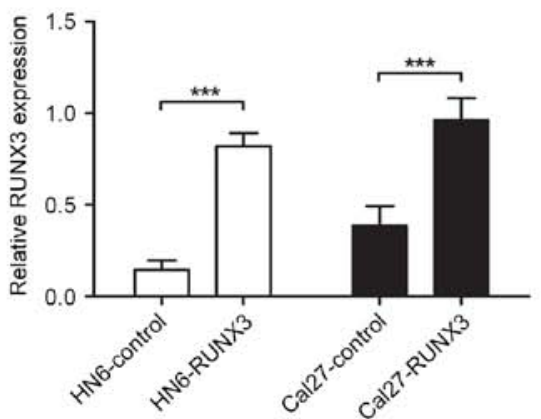

Cal27-RUNX3
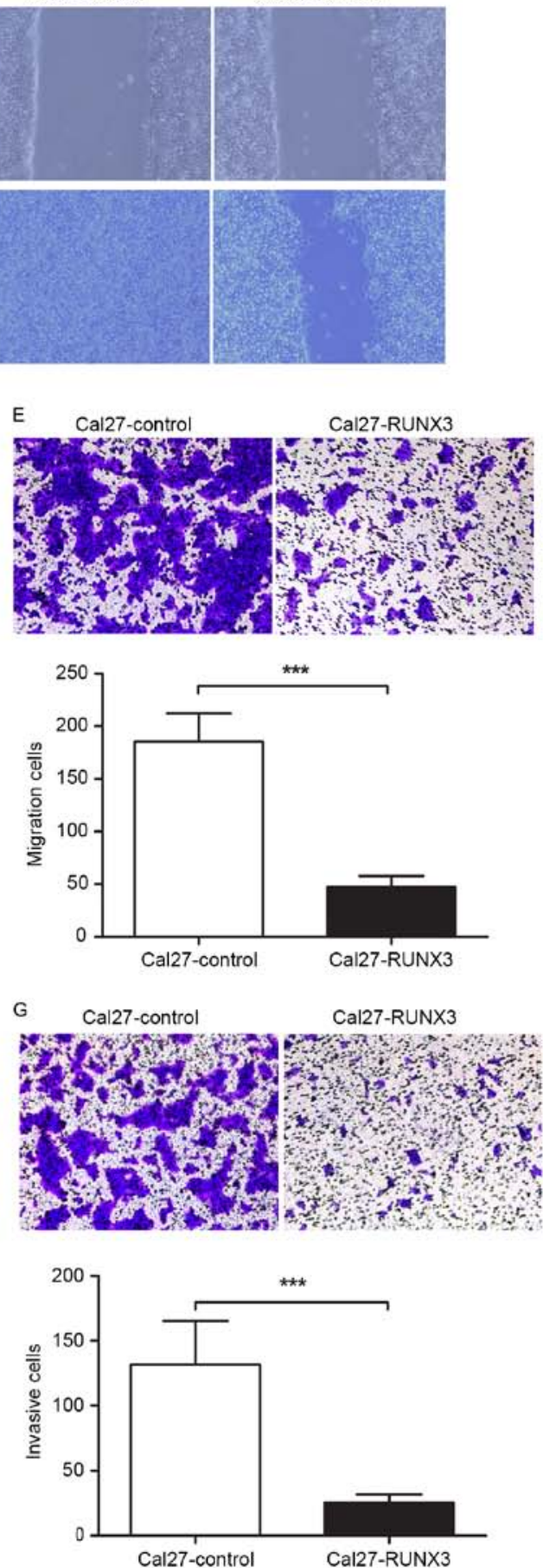

Figure 2. RUNX3 restoration suppresses OSCC cell migration and invasion. (A and B) Western blot analysis of the relative protein levels of RUNX3 in OSCC cell lines HN6 and Cal27 transfected with pFlag-RUNX3 or pFlag-control. RUNX3 expression was significantly enhanced after 24-h transfection. Actin was used as a control for protein loading. (C and D) Wound healing and migration assays were performed after RUNX3 restoration in HN6 and Cal27 cells. (E and F) Invasion assay was performed after RUNX3 restoration in HN6 and Cal27 cells. The data are presented as mean \pm SD for triplicate determinations. ${ }^{* * * *} \mathrm{P}<0.01$. 
A

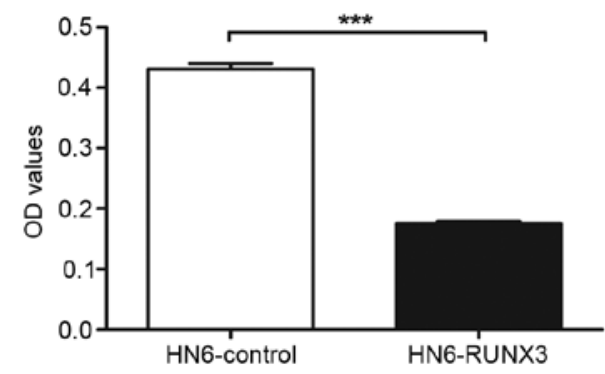

C HN6-control
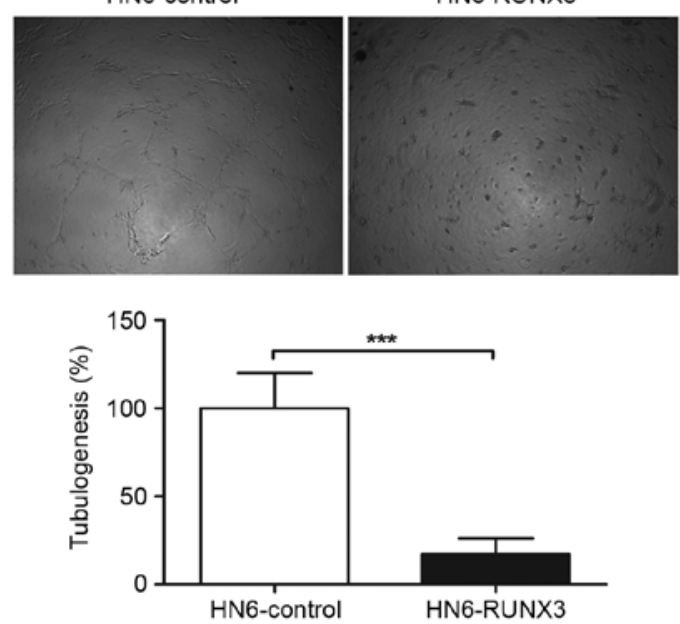

B

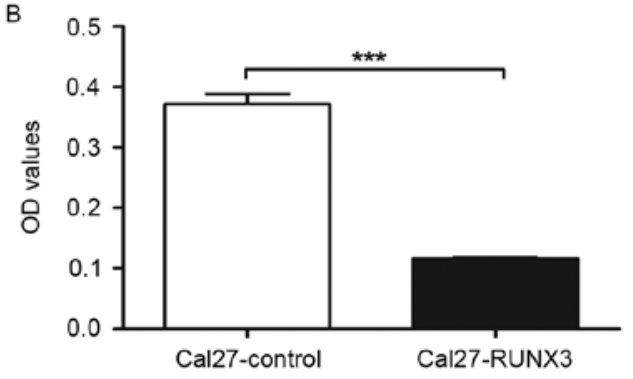

D
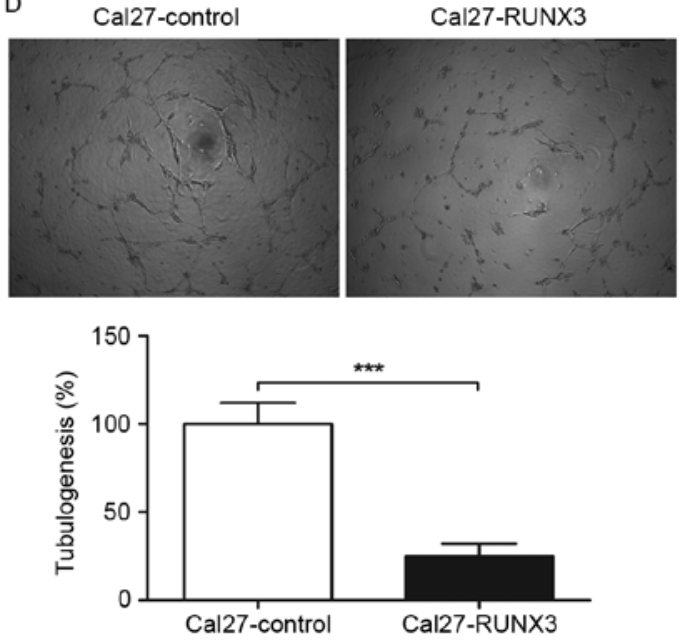

Figure 3. Reduction of angiogenesis in HN6 and Cal27 cells by restoration of RUNX3 expression. (A and B) CCK-8 cell proliferation assay was performed to detect the HUVEC proliferation cultured with the supernatant from HN6 and Cal27 cells transfected with RUNX3 or vehicle control. (C and D) Representative images of HUVEC tube formation cultured with the supernatant from HN6 and Cal27 cells transfected with RUNX3 or vehicle control. Control cell cultures were given arbitrary percentage values of 100 . All experiments were carried out in triplicate. Data are shown as mean \pm SD. ${ }^{* * *} \mathrm{P}<0.01$.

cell invasive ability of HN6 and Cal27 cells in Matrigel-coated Transwell (Fig. 2F and G).

Reduction of HUVEC proliferation and tube formation with conditioned medium from RUNX3 transfected OSCC cells in vitro. To further determine the effect of restored RUNX3 expression on angiogenic potential of human OSCC cells, we used the supernatant of HN6 and Cal27 cells as conditioned medium to culture the endothelial cell line HUVECs. We observed that conditioned medium from HN6 and Cal27 cells transfected with pFlag-RUNX3 inhibited proliferation of HUVECs compared with those of pFlag-control transfected cells (Fig. 3A and B). In tube formation assay, the average tube length of HUVECs cultured with supernatant from pFlag-RUNX3 transfected HN6 and Cal27 cells was significantly decreased in contrast with that of vehicle control, as shown in Fig. 3C and D.

Suppression of MMP-9 and VEGF expression and secretion in RUNX3 transfected OSCC cells. To investigate the mechanisms of RUNX3 regulating invasion and angiogenesis, we performed western blotting and ELISA to detect the MMPs and VEGF levels in OSCC. We found expression of MMP-9 and VEGF protein was downregulated significantly in HN6 and Cal27 cells transfected with pFlag-RUNX3 (Fig. 4A-C). Also, the restoration of RUNX3 in HN6 and Cal27 cells led to a significant reduction of MMP-9 and VEGF secretion in conditioned medium (Fig. 4D and E).

\section{Discussion}

This study examined the status of RUNX3 expression in OSCC by TMA technology and found RUNX3 was inactivated in 139 of 232 OSCC specimens. Among these 139 cases, RUNX3 protein could be underexpressed (54 cases) or mislocalized in the cytoplasm (85 cases). RUNX3 was frequently inactivated by two mechanisms of protein mislocalization and promoter hypermethylation, which had been proved in many cancers including OSCC $(7,10,18-20)$. In OSCC, Gao et al (18) reported that $30 \%$ OSCC specimens presented with exclusive RUNX3 cytoplasmic retention, and 55.3\% showed underexpression of RUNX3. Also, both of these aberrant RUNX3 statues were correlated with tumor differentiation, but not with local lymph node metastasis (18). Supic et al found that RUNX3 gene promoter hypermethylation was significantly associated with lymph node involvement and tumor stage, but not with the overall survival of tongue carcinoma (20). In the present study, we analyzed these two types together as negative expression of RUNX3, which was significantly correlated with tumor size, lymph node metastasis and clinical stage, but not with tumor differentiation. All these findings strongly supported the notion that RUNX3 acted as a tumor suppressor in OSCC.

Loss of RUNX3 expression was proved to worsen poor survival in breast, gastric cancer, and esophageal cancer $(11,21,22)$. These studies indicated that the loss of RUNX3 expression may contribute to tumor metastasis. 
A
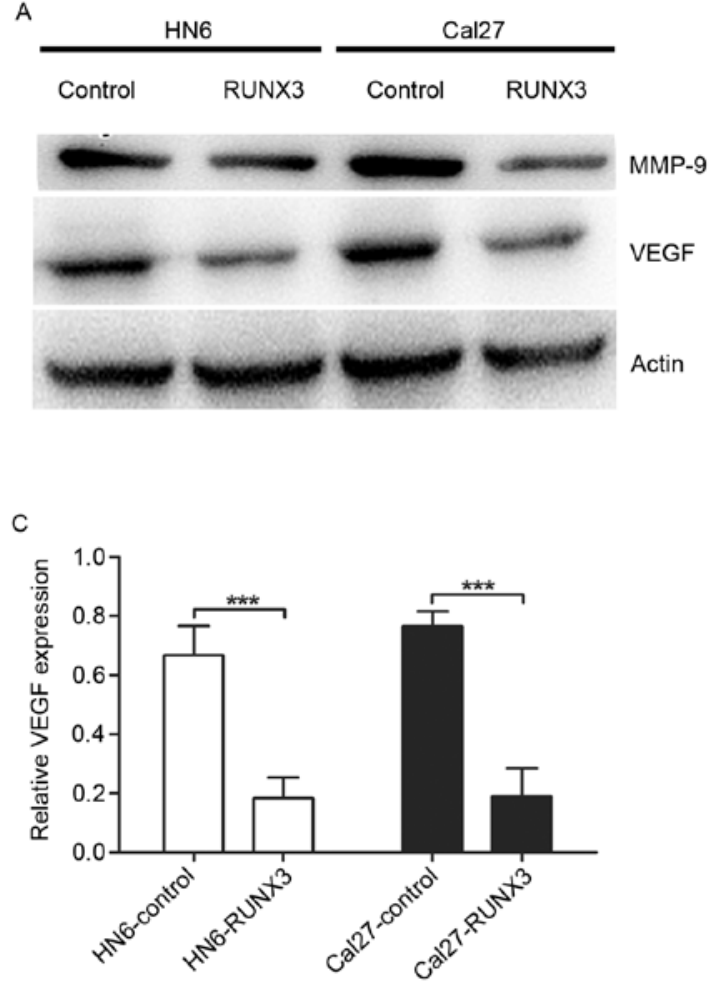

B

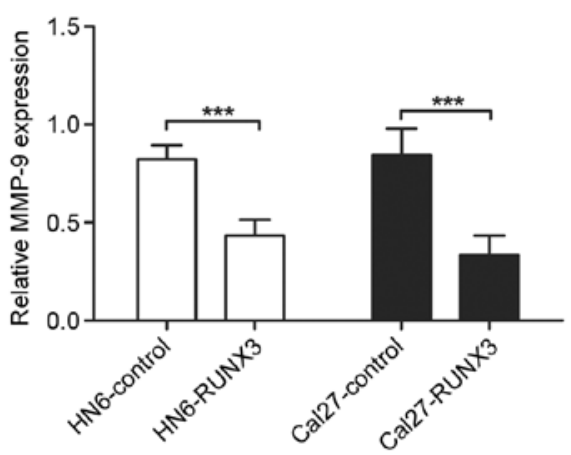

D

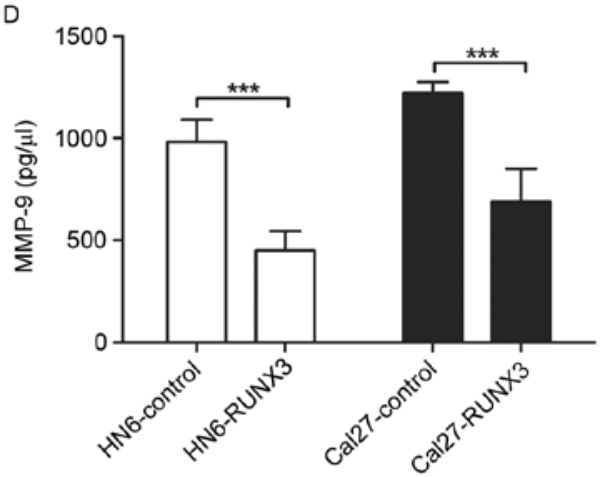

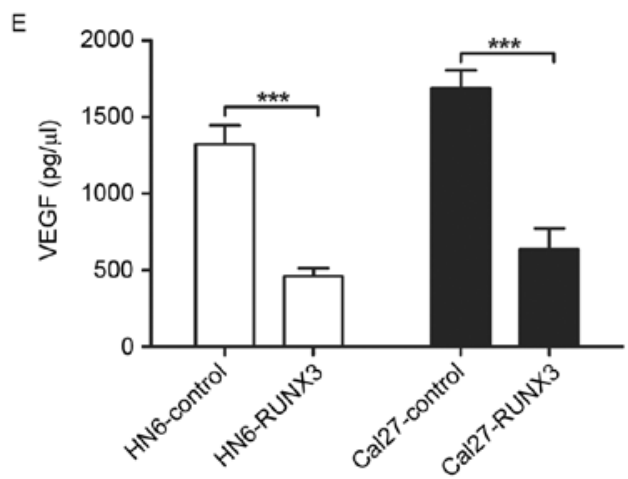

Figure 4. RUNX3 inhibits the expression and secretion of MMP-9 and VEGF in OSCC cells. (A-C) Western blot analysis of the relative protein levels of MMP-9, VEGF and actin in HN6 and Cal27 cells transfected with RUNX3 or vehicle control group. (D and E) ELISA analysis of the secretion of MMP-9 and VEGF in RUNX3 restoration and control group. All experiments were carried out in triplicate. Data are shown as mean \pm SD. ${ }^{* * * *} \mathrm{P}<0.01$.

In the present study, negative expression of RUNX3 was associated with some clinical aspects which were crucial to OSCC prognosis. Based on the follow-up analysis, the data of 110 cases with at least 5-year follow-up demonstrated that negative expression of RUNX3 worsened the patient survival. Therefore, RUNX3 could be a potential prognostic factor of OSCC.

Cumulated studies proved that the restoration of RUNX3 could inhibit malignant behavior of various cancer cells $(12,13)$. RUNX3 is a downstream target of transforming growth factor- $\beta$ (TGF- $\beta$ ) mediated tumor suppressor pathway, the key regulator of cellular proliferation, invasion and migration in a variety of different cancer types (23). Moreover, RUNX3 is a negative modulator of Wnt (wingless type)/ $\beta$-catenin signaling pathway, which is involved in the cancer initiation and malignant transformation in a wide range of human cancers, including OSCC (24). In our previous studies, we reported that attenuation of RUNX3 expression could suppress the cell migration and invasion ability in breast, renal tumor and glioma $(11,14,25)$. In the present study, we confirmed that these effects of RUNX3 restoration were equally important on OSCC cell lines. The abilities of migration and invasion were significantly inhibited by reintroduction of RUNX3 in HN6 and Cal27 cells.

There are reports that TGF- $\beta$ signaling pathway could influence cancer cells migration and invasion by regulating matrix metalloproteinases (MMPs) such as MMP-2 and MMP-9 (26). Being a key downstream molecule of TGF- $\beta$ signaling pathway, RUNX3 may have the potential role in controlling MMPs which has been reported to participate closely in tumor progression (27). RUNX3 overexpression inhibited cell migration and invasion by downregulation of MMP-9 expression in human esophageal squamous cell carcinoma (13). We previously determined the relationship between RUNX3 and MMP-2 in glioma and breast cancer $(11,14)$, as well as MMP-9 in renal cancer cells (25). Here, we found downregulation of MMP-9 protein and decreased secretion of MMP-9 in HN6 and Cal27 cells 
transfected with RUNX3. Our data indicated that RUNX3 may suppress invasion and migration through downregulating MMP-9 expression and secretion in OSCC cell lines in vitro.

Besides the inhibition of invasion and migration, RUNX3 played the role of tumor suppressor through antiangiogenesis $(12,25,28)$. In the present study, we found that supernatant from HN6 and Cal27 cells transfected with RUNX3 had less ability to induce HUVEC proliferation and tube formation compared with that from vehicle control. These results implied that the angiogenic potential of OSCC cells was reduced by RUNX3 restoration. Furthermore, we detected the expression and secretion of VEGF, which had been proved to be a vital angiogenic factor of tumor blood vessel formation in solid tumors (29). The relationship between RUNX3 and VEGF was determined in previous studies $(12,25)$. In western blotting and ELISA analysis, our results also demonstrated that VEGF expression and secretion was decreased by restoration of RUNX3 in OSCC cell lines in vitro.

Recently, the role of RUNX3 as an oncogene was promoted in skin cancer and HNSCC $(15,16,30)$. Tsunematsu et al presumed this distinct oncogenic role of RUNX3 attributed to the pathogenesis of skin cancer and HNSCC, both of which arose from squamous epithelium. Also, the functional mutation or protein mislocalization of RUNX3 was not found in HNSCC cells (15). However, hypermethylation of RUNX3 gene promoter was observed in $35 \%$ of tongue cancer, one type of OSCC origin from squamous epithelium, and was significantly associated with lymph node involvement and tumor stage (20). In addition, a reduced expression and protein mislocalization of RUNX3 were observed in esophageal squamous cell carcinoma $(13,31)$. Our TMA results demonstrated that protein mislocalization of RUNX3 was frequent in OSCC tissues. These studies indicated that the function of RUNX3 may be different even in the same pathogenesis of cancer cells. Further experiments are required to clarify this phenomenon.

In conclusion, our results verified that reduced RUNX3 expression significantly correlated with tumor size, local lymph node metastasis and clinical stage, and predicted poor prognosis in OSCC. RUNX3 regulated OSCC cancer cell migration, invasion and angiogenesis through suppressing MMP-9 and VEGF expression and activity. Our clinical and mechanistic data indicated that RUNX3 played a tumor suppressor role in OSCC, and targeting of RUNX3 pathway could be a potential therapy for OSCC.

\section{Acknowledgements}

This study was supported by the National Natural Science Foundation of China (81672678), The Project Funded by the Priority Academic Program Development of Jiangsu Higher Education Institutions (PAPD, 2014-37), The Project Funded by Jiangsu Provincial Commission of Health and Family Planning (Z201511), and The Open Project of Jiangsu Key Laboratory of Oral Diseases (JSKLOD-KF-1501).

\section{References}

1. Warnakulasuriya S: Global epidemiology of oral and oropharyngeal cancer. Oral Oncol 45: 309-316, 2009.
2. Lambert R, Sauvaget $C$, de Camargo Cancela $M$ and Sankaranarayanan R: Epidemiology of cancer from the oral cavity and oropharynx. Eur J Gastroenterol Hepatol 23: 633-641, 2011.

3. Weatherspoon DJ, Chattopadhyay A, Boroumand S and Garcia I: Oral cavity and oropharyngeal cancer incidence trends and disparities in the United States: 2000-2010. Cancer Epidemiol 39: 497-504, 2015.

4. Ghantous Y, Yaffi V and Abu-Elnaaj I: Oral cavity cancer: Epidemiology and early diagnosis. Refuat Hapeh Vehashinayim 32: 55-63, 71, 2015 (In Hebrew).

5. Ito Y: Oncogenic potential of the RUNX gene family: 'Overview'. Oncogene 23: 4198-4208, 2004.

6. Tonomoto Y, Tachibana M, Dhar DK, Onoda T, Hata K, Ohnuma H, Tanaka T and Nagasue N: Differential expression of RUNX genes in human esophageal squamous cell carcinoma: Downregulation of RUNX3 worsens patient prognosis. Oncology 73: 346-356, 2007.

7. Ito K, Liu Q, Salto-Tellez M, Yano T, Tada K, Ida H, Huang C, Shah N, Inoue M, Rajnakova A, et al: RUNX3, a novel tumor suppressor, is frequently inactivated in gastric cancer by protein mislocalization. Cancer Res 65: 7743-7750, 2005.

8. Soong R, Shah N, Peh BK, Chong PY, Ng SS, Zeps N, Joseph D, Salto-Tellez M, Iacopetta B and Ito Y: The expression of RUNX3 in colorectal cancer is associated with disease stage and patient outcome. Br J Cancer 100: 676-679, 2009.

9. Yu GP, Ji Y, Chen GQ, Huang B, Shen K, Wu S and Shen ZY: Application of RUNX3 gene promoter methylation in the diagnosis of non-small cell lung cancer. Oncol Lett 3: 159-162, 2012.

10. Lau QC, Raja E, Salto-Tellez M, Liu Q, Ito K, Inoue M, Putti TC, Loh M, Ko TK, Huang C, et al: RUNX3 is frequently inactivated by dual mechanisms of protein mislocalization and promoter hypermethylation in breast cancer. Cancer Res 66: 6512-6520, 2006.

11. Bai J, Yong HM, Chen FF, Song WB, Li C, Liu H and Zheng JN: RUNX3 is a prognostic marker and potential therapeutic target in human breast cancer. J Cancer Res Clin Oncol 139: 1813-1823, 2013.

12. Kim BR, Kang MH, Kim JL, Na YJ, Park SH, Lee SI, Kang S, Joung SY, Lee SY, Lee DH, et al: RUNX3 inhibits the metastasis and angiogenesis of colorectal cancer. Oncol Rep 36: 2601-2608, 2016.

13. Chen H, Wang Z, Wang S, Zhang Z and Shi S: Effect and mechanism of RUNX3 gene on biological characteristics of human esophageal squamous cell carcinoma (ESCC). Med Oncol 32: 357, 2015.

14. Mei PJ, Bai J, Liu H, Li C, Wu YP, Yu ZQ and Zheng JN: RUNX3 expression is lost in glioma and its restoration causes drastic suppression of tumor invasion and migration. J Cancer Res Clin Oncol 137: 1823-1830, 2011.

15. Tsunematsu T, Kudo Y, Iizuka S, Ogawa I, Fujita T, Kurihara H, Abiko Y and Takata T: RUNX3 has an oncogenic role in head and neck cancer. PLoS One 4: e5892, 2009.

16. Kudo Y, Tsunematsu T and Takata T: Oncogenic role of RUNX3 in head and neck cancer. J Cell Biochem 112: 387-393, 2011.

17. Tanji Y, Osaki M, Nagahama Y, Kodani I, Ryoke K and Ito H: Runt-related transcription factor 3 expression in human oral squamous cell carcinomas; implication for tumor progression and prognosis. Oral Oncol 43: 88-94, 2007.

18. Gao F, Huang C, Lin M, Wang Z, Shen J, Zhang H, Jiang L and Chen Q: Frequent inactivation of RUNX3 by promoter hypermethylation and protein mislocalization in oral squamous cell carcinomas. J Cancer Res Clin Oncol 135: 739-747, 2009.

19. Subramaniam MM, Chan JY, Soong R, Ito K, Ito Y, Yeoh KG, Salto-Tellez M and Putti TC: RUNX3 inactivation by frequent promoter hypermethylation and protein mislocalization constitute an early event in breast cancer progression. Breast Cancer Res Treat 113: 113-121, 2009.

20. Supic G, Kozomara R, Jovic N, Zeljic K and Magic Z: Hypermethylation of RUNX3 but not WIF1 gene and its association with stage and nodal status of tongue cancers. Oral Dis 17: 794-800, 2011

21. Shi M, Wang Z, Liu XY and Chen D: Inactivation of RUNX3 predicts poor prognosis in esophageal squamous cell carcinoma after Ivor-Lewis esophagectomy. Med Oncol 31: 309, 2014.

22. Cheng HC, Liu YP, Shan YS, Huang CY, Lin FC, Lin LC, Lee L, Tsai CH, Hsiao M and Lu PJ: Loss of RUNX3 increases osteopontin expression and promotes cell migration in gastric cancer. Carcinogenesis 34: 2452-2459, 2013.

23. Subramaniam MM, Chan JY, Yeoh KG, Quek T, Ito K and Salto-Tellez M: Molecular pathology of RUNX3 in human carcinogenesis. Biochim Biophys Acta 1796: 315-331, 2009. 
24. Romana PG: WITHDRAWN: Cell alterations and molecular mechanisms in oral carcinogenesis. Int J Oral Maxillofac Surg: Aug 4, 2010 (Epub ahead of print).

25. Chen F, Bai J, Li W, Mei P, Liu H, Li L, Pan Z, Wu Y and Zheng J: RUNX3 suppresses migration, invasion and angiogenesis of human renal cell carcinoma. PLoS One 8: e56241, 2013.

26. Krstic J and Santibanez JF: Transforming growth factor-beta and matrix metalloproteinases: Functional interactions in tumor stroma-infiltrating myeloid cells. Sci World J 2014: 521754, 2014.

27. Martin MD and Matrisian LM: The other side of MMPs: Protective roles in tumor progression. Cancer Metastasis Rev 26: 717-724, 2007.

28. Peng Z, Wei D, Wang L, Tang H, Zhang J, Le X, Jia Z, Li Q and Xie K: RUNX3 inhibits the expression of vascular endothelial growth factor and reduces the angiogenesis, growth, and metastasis of human gastric cancer. Clin Cancer Res 12: 6386-6394, 2006.
29. Cao EG, Wang E, Pal K, Dutta SK, Bar-Sagi D and Mukhopadhyay D: VEGF exerts an angiogenesis-independent function in cancer cells to promote their malignant progression. Cancer Res 72: 3912-3918, 2012.

30. Lee JH, Pyon JK, Kim DW, Lee SH, Nam HS, Kang SG, Kim CH, Lee YJ, Chun JS and Cho MK: Expression of RUNX3 in skin cancers. Clin Exp Dermatol 36: 769-774, 2011.

31. Hiramatsu T, Osaki M, Ito Y, Tanji Y, Tokuyasu N and Ito $\mathrm{H}$ : Expression of RUNX3 protein in human esophageal mucosa and squamous cell carcinoma. Pathobiology 72: 316-324, 2005. 